\title{
EFICACIA DEL PROGRAMA NACIONAL DE INGLÉS 2015-2025 IMPLEMENTADO EN LAS INSTITUCIONES EDUCATIVAS DEL DEPARTAMENTO NORTE DE SANTANDER
}

\section{EFFECTIVENESS OF THE NATIONAL ENGLISH PROGRAM 2015-2025 IMPLEMENTED IN THE EDUCATIONAL INSTITUTIONS OF THE DEPARTMENT OF NORTE DE SANTANDER}

Jessica Jessenia Reales Roa ${ }^{1}$

\section{Marling Carolina Cordero²}

\section{Raúl Prada Núñez ${ }^{3}$}

UFPS

\section{RESUMEN}

La presente investigación concentró sus esfuerzos en identificar las fortalezas, debilidades, amenazas del Plan Nacional de Inglés 2015-2025 implementado en las

\footnotetext{
$1 \quad$ Administradora de empresas de la Universidad Francisco Paula Santander. Correo electrónico: jessenia12 2@hotmail.com 0000-0001-9977-7183.

2 Magíster en Gerencia de Empresas, mención Finanzas por la Universidad Nacional y Experimental del Táchira. Docente investigador de la Universidad Francisco de Paula Santander. Correo electrónico: marlingcarolinacd@ ufps.edu.co. Orcid: 0000-0002-2913-5588

3 Magíster en Ingeniería de Análisis de Datos, Mejora de Procesos y Toma de decisiones por la Universidad Politécnica de Valencia (España). Docente investigador de la Universidad Francisco de Paula Santander. Correo electrónico: raulprada@ufps.edu.co. Orcid: 0000-00016145-1786.
}

instituciones educativas del departamento de Norte de Santander., para lo cual se llevó a cabo una investigación bajo un enfoque cuantitativo en el cual se usó la encuesta como técnica de recolección de información con preguntas cerradas en una escala de 1 a 5 , dónde 5 es excelente y 1 es deficiente, aplicadas a los docentes del área de inglés de instituciones de educación básica secundaria de los municipios de Cúcuta, Villa del Rosario y Los Patios; Los resultados se analizaros a través de gráficas y tabla de ponderación con la que se construyó una matriz DOFA con la que se establecieron las fortalezas y debilidades del Programa en el departamento al igual que permitió formular estrategias de gestión que pueden ser aplicadas 
para mejorar los procesos durante la ejecución del plan. La investigación llegó a la conclusión de que es necesario establecer indicadores de gestión y mejorar la interoperabilidad entre las instituciones educativas y las gubernamentales, así como la integración de los docentes en los procesos de determinación de los temas y herramientas a usar durante las jornadas de capacitación.

\section{PALABRAS CLAVES}

Eficacia, Programa Nacional de Inglés 20152025, inglés.

\section{ABSTRACT}

The objective of this research was to identify the National English Plan 2015-2025's strengths, weaknesses and dangers, which was implemented in the educative institutions from North of Santander, for which research was conducted under a quantitative approach in which the survey was used as an information$\begin{array}{llll}3 & 0 & 2\end{array}$. gathering technique with closed questions on a scale of 1 to 5 , where 5 is excellent and 1 is poor, applied to teachers in the English area of secondary basic education institutions of the municipalities of Cúcuta, Villa del Rosario and Los Patios; The results were analyzed through graphs and weighting table with which a DOFA matrix was built with which the strengths and weaknesses of the Program were established in the department as well as allowed to formulate management strategies that can be applied to improve processes during the implementation of the plan. The research concluded that management indicators need to be established and interoperability improved between educational and government institutions and the integration of teachers into the processes of determining the topics and tools to be used during training days.

\section{KEYWORDS}

Efficacy, National English Program 2015-2025, English.

\section{INTRODUCCIÓN}

A nivel mundial, la sociedad contemporánea se encuentra sumergida en la modalidad de tecnologías de la información dentro de un contexto de globalización, por ende, el aprendizaje de las lenguas es un requisito primordial. El dominio de una segunda lengua implica mayores posibilidades de insertarse adecuadamente en la dinámica de la globalización, por lo que quienes la posean harán uso de una importante herramienta para acceder a la cultura de la modernidad. La importancia, entonces, de una segunda lengua, se presenta como una necesidad vital y surge lo que se conoce como bilingüismo.

Seguidamente de institucionalizar el bilingüismo, el gobierno nacional, por medio del Ministerio de Educación, ha ofrecido varios planes para mejorar el nivel de inglés en los estudiantes de educación básica secundaria, docentes y educandos de Licenciatura en Idiomas, entre las más reciente el Programa Nacional de Inglés 2015 - 2025 (Ministerio Educación, 2014).

Es por esto que el implementar políticas públicas para que se desarrollen programas que fortalezcan los medios de aprendizaje en las instituciones se vuelve algo necesario en Colombia, y al estudiar estos programas requiere enfocarse en el Plan Nacional de Inglés very well (Ministerio Educación, 2014) para diagnosticar su eficacia a través de poder conocer sus debilidades como programa en el acceso, conocimiento a los profesores que son el primer recurso humano con el que se lleva a cabo este plan, y la capacitación de ellos mismos. La oportunidad que abre la puerta al exterior, sus políticas públicas, y el interés que todos los colombianos manejen este idioma, 
la oportunidad de expandir el mercado y mirar otros territorios es el entusiasmo de muchos egresados al poder dominar esta segunda lengua, y las oportunidades de un comercio exterior al empresario de Colombia y en el caso particular del Departamento Norte de Santander. Conociendo el programa que lleva el plan nacional de inglés su fortaleza es la aprobación y conocimiento de los docentes de Inglés, y esto es algo que fortalece al programa, dado que sus expectativas son grandes, pero una debilidad es llevarse a cabo en los estudiantes y que ellos puedan seguir desarrollando y avanzando en el dominio de una segunda lengua.

De acuerdo con esta problemática social que abre o cierra puertas, el reto es indagar como se aporta al mejoramiento del plan que ya está en ejecución, considerando cuatro escenarios de análisis frente a las Fortalezas y Oportunidades, Debilidades y Oportunidades, Fortalezas y Amenazas, Debilidades y Amenazas.

De esta manera se llega a concluir que al revisar el indicador de ¿cómo? puede ser eficaz en el cumplimiento de los resultados que espera en unos años el programa que se está llevando a cabo en el municipio de San José de Cúcuta. Con el desarrollo de un análisis cuantitativo a través de la aplicación de una encuesta a los profesores de Inglés de instituciones públicas de la ciudad, conociendo este diagnóstico se esperaría que se diseñen una serie de estrategias tendientes a garantizar la efectividad del programa. Se quiere con este trabajo que usted como lector pueda conocer las bases y hacia donde desea llegar el Plan Nacional de Inglés, la invitación es que se pueda sumergir en el criterio de esta investigación que fue llevada gracias al apoyo de la Secretaria de Educación Municipal, al apoyo de un grupo de tutores de la Fundación de Estudios Superiores Comfanorte (FESC) y que contó con el acompañamiento de profesionales de otras instituciones de educación superior de la ciudad.

\section{METODOLOGÍA}

\section{Enfoque, nivel y diseño de la investigación.}

Esta investigación se enmarca dentro de las características del enfoque cuantitativo a nivel descriptivo, puesto que se apoya en la recolección de datos para validar ciertos supuestos por medio del cálculo de estadísticos descriptivos, que en este caso, el objetivo es identificar la percepción de eficacia del Plan Nacional de Inglés en opinión de los docentes de Instituciones Educativas del Departamento de Norte de Santander y describir el enfoque resultante del estado actual del dicho plan en el departamento en el periodo 2015-2019. Por todo lo mencionado, los datos fueron recolectados de la fuente primaria (Hernández et al., 2014).

Población y muestra. Según Lepkowski (citado por Hernández et al., 2014), la población o universo es el conjunto de todos los elementos o personas que cumplen con ciertos criterios de selección. A partir de esa definición, se debe mencionar que en el Departamento de Norte de Santander hay 40 municipios, en los que se encuentran, según Serrano (2020):

211 establecimientos educativos, 109 Centros Educativos Rurales, 102 Instituciones Educativas (43 Rurales- 59 urbanas) con matrícula total en el SIMAT de 177.804 estudiantes de educación preescolar básica y media. Por su parte Cúcuta, capital del departamento posee 61 establecimientos educativos oficiales y 219 sedes educativas de las cuales con corte a 2019 según SIMAT 133.501 estudiantes en el sector oficial 34.007 del sector privado para un total de 167.508 (p.26). 
Para el desarrollo de esta investigación se recurrió al muestreo no probabilístico, bajo la técnica de muestreo intencional, dado que de los 211 establecimientos educativos del Departamento y los 61 establecimientos de la ciudad de Cúcuta (en total 272 instituciones educativas), fueron seleccionados 50 instituciones públicas de los tres grandes municipios que conforman el área metropolitana de Cúcuta. La conformación de la muestra se realizó mediante la designación proporcional en función del número de instituciones educativas en cada uno de ellos, así: a Cúcuta se le asignó el $50 \%$, Villa del Rosario el $30 \%$ y Los Patios el $20 \%$. Para la vinculación en la muestra se siguieron los siguientes criterios de selección: a) estar ubicado en los municipios de observación; b) al menos tener dos docentes de inglés; c) ofertar los diversos grados de la Educación Básica; y d) el rector de la institución se comprometa a facilitar el acceso a los informantes. De esta forma, se conformó una muestra de 60 instituciones educativas, y de cada una de ellas se seleccionaron dos docentes. En la tabla 1 se resume en cifras el proceso de muestreo.

Tabla 1. Distribución de la muestra aplicada por municipios

\begin{tabular}{cccccc}
\hline Municipio & $\begin{array}{c}\text { Población (No. } \\
\text { De Instituciones } \\
\text { educativas) }\end{array}$ & Muestra & $\begin{array}{c}\text { Tamaño de } \\
\text { la muestra } \\
\text { (Instituciones } \\
\text { Educativas) }\end{array}$ & $\begin{array}{c}\text { Tamaño de la } \\
\text { muestra } \\
\text { (docentes) }\end{array}$ & $\begin{array}{c}\text { Criterio de } \\
\text { Selección }\end{array}$ \\
\hline $\begin{array}{l}\text { Cúcuta } \\
\text { Villa del } \\
\text { Rosario }\end{array}$ & 61 & $50 \%$ & 30 & 60 & Docentes \\
$\begin{array}{c}\text { Los Patios } \\
\text { TOTAL }\end{array}$ & 211 & $30 \%$ & 20 & 40 & de \\
(departamento) & $20 \%$ & 10 & 20 & Bducación \\
Búsa
\end{tabular}

Fuente: Elaboración Propia (2021)

\section{Técnicas e instrumentos de recolección} de información. Para evaluar la eficacia del Plan Nacional de Inglés 2015-2015 en el Departamento de Norte de Santander se utilizó un cuestionario con escala de Likert (Excelente - 5, Bueno - 4, Regular - 3, Malo - 2, Deficiente 1) para identificar la percepción de los docentes frente a las diferentes actividades que conforman el plan y los resultados obtenidos en los cinco años en que ha estado en ejecución.

\section{RESULTADOS Y DISCUSIÓN}

Identificación de las fortalezas, debilidades, amenazas del Plan Nacional de Inglés 20152025 implementado en las instituciones educativas del departamento de Norte de Santander.
Para identificar las fortalezas, debilidades y amenazas del Plan Nacional de Inglés se determinaron las medias de cada pregunta y el resultado se clasificó según la siguiente escala valorativa: a) Entre 4.5 a 5.0 desempeño excelente; b) Entre 3.5 a 4.4 desempeño bueno; c) Entre 2.5 a 3.4 desempeño regular; d) Entre 1.5 a 2.4 desempeño bajo; e) Entre 0.1 a 1.4 desempeño deficiente. A partir de estos resultados de las medias se construyó la tabla 2 que corresponde a una equivalencia en función de una matriz DOFA. 
Tabla 2: Matriz DOFA Programa Nacional de Inglés en el Departamento de Norte de Santander

FORTALEZAS

DEBILIDADES
Grado de conocimiento de los docentes sobre qué es el Programa Nacional de Inglés.

Buena percepción sobre la meta de mejorar el nivel del $50 \%$ de los estudiantes al año 2025 en las Pruebas Saber 11.

Aprobación de la intervención del modelo pedagógico en el proceso de enseñanza del inglés.

Los docentes aprueban el uso de tecnologías durante el proceso de enseñanza del inglés.

Apropiación del Programa Nacional de Inglés dentro del PEI de las instituciones.

Apropiación del Programa Nacional de Inglés dentro del área de Inglés de las instituciones educativas y planes de aula.

El dominio de los temas por parte del capacitador, y su capacidad pedagógica.

La aplicabilidad de los conocimientos adquiridos en el proceso de enseñanza del idioma.

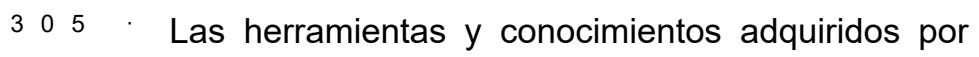
los docentes para su uso dentro de los procesos de enseñanza y aprendizaje.
El acceso a infraestructura física y tecnológica, dentro del marco del Programa Nacional de Inglés.

El número de capacitaciones a las que tienen acceso los docentes.

El contenido de las capacitaciones y su orientación a resolver las necesidades de los docentes.

La profundidad del contenido de las capacitaciones.

La estructuración y el material usado en las capacitaciones a docentes.

La duración de las capacitaciones.

\section{AMENAZAS}

OPORTUNIDADES

Socialización dentro de las instituciones educativas, Infraestructura tecnológica puede ser usada de las capacitaciones docentes y de los resultados de las mismas en el marco del programa.

El trabajo realizado conjunto entre la institución educativa y los gobiernos locales y departamentales en el marco del Programa Nacional del Inglés.

Aplicación, por parte de los docentes, de lo aprendido para el fortalecimiento del idioma inglés.

La Ley 1651 de 2013, Ley de Bilingüismo, abre la puerta para que se puedan destinar recursos de SGP para la generación de programas complementarios para lograr los objetivos el Plan Nacional de Inglés. en las capacitaciones para mejorar el proceso de enseñanza del Inglés.

Fuente. Elaboración propia 
Determinación de la percepción de los docentes de las Instituciones Educativas del departamento de Norte de Santander sobre el Plan Nacional de Inglés 2015-2025.

A partir de los resultados de la aplicación del instrumento se determina la percepción que tienen los docentes sobre el Programa Nacional de Inglés en el Departamento de Norte de Santander. Entre los resultados más destacados se encuentra el conocimiento general del programa por parte de los docentes; de los 120 docentes encuestados el 69,39\% afirma que poseen buen conocimiento sobre el programa, en contraste con el 10,20\% quienes aseguran desconocer de la existencia del mismo y su aplicación en la institución educativa (ver Gráfico 1). Estos resultados reflejan fallas en la comunicación con las instituciones educativas y los ejecutores del programa, lo cual impide la integración de docentes generando una brecha que podría hacerse más grande con el paso del tiempo y podría poner en riesgo la obtención de resultados toda vez que el programa tiene sus metas a largo plazo.

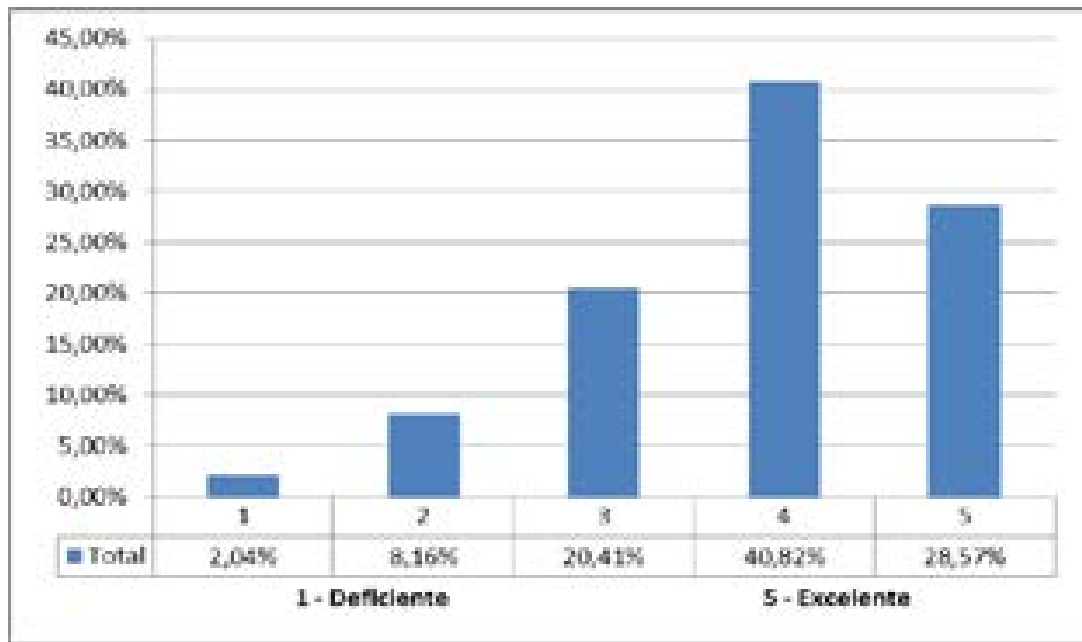

Gráfico 1. Grado de conocimiento de los docentes sobre el Programa Nacional de Inglés 2019- 2025. Fuente. Elaboración Propia.

Al indagar entre los docentes sobre el nivel de satisfacción con el programa adelantando y el modelo pedagógico implementado, se pudo identificar que $79,59 \%$ se muestran satisfechos con las estrategias de enseñanza adelantadas y como ellas han ido influyendo en el proceso de aprendizaje, mientras que en el porcentaje restante $16,33 \%$ tienen reservas frente a la intervención del modelo educativo que busca mejorar la enseñanza de inglés en las instituciones educativas públicas del país (ver Gráfico 2). 


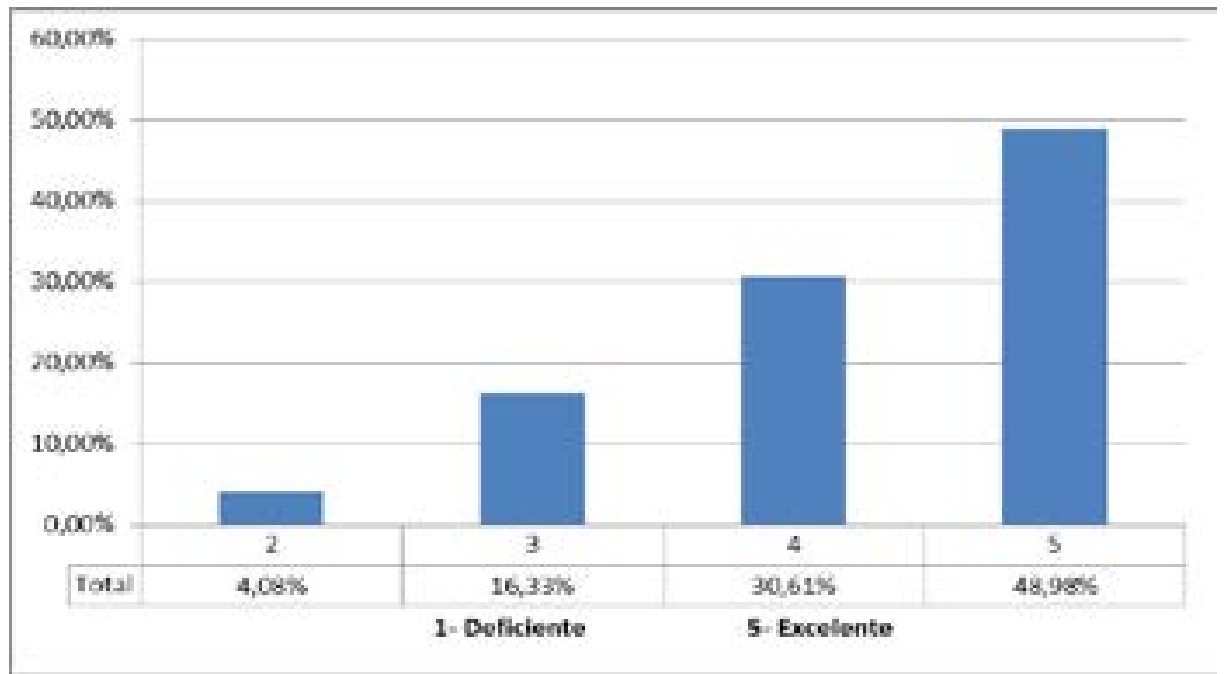

Gráfico 2. Percepción de la intervención del modelo pedagógico.

Fuente. Elaboración Propia.

Es de resaltar que para el $55,09 \%$ de los docentes encuestados el acceso al Programa Nacional de Inglés, por parte de las instituciones educativas, tiene aspectos por mejorar (ver Gráfico 3). Este resultado es un indicador de que el programa podría no estar llegando de forma adecuada a todas las instituciones educativas y los docentes no se están sintiendo participes de él, o hace falta mayor cubrimiento de los planes $\mathrm{y}$ beneficios a todas las instituciones educativas.

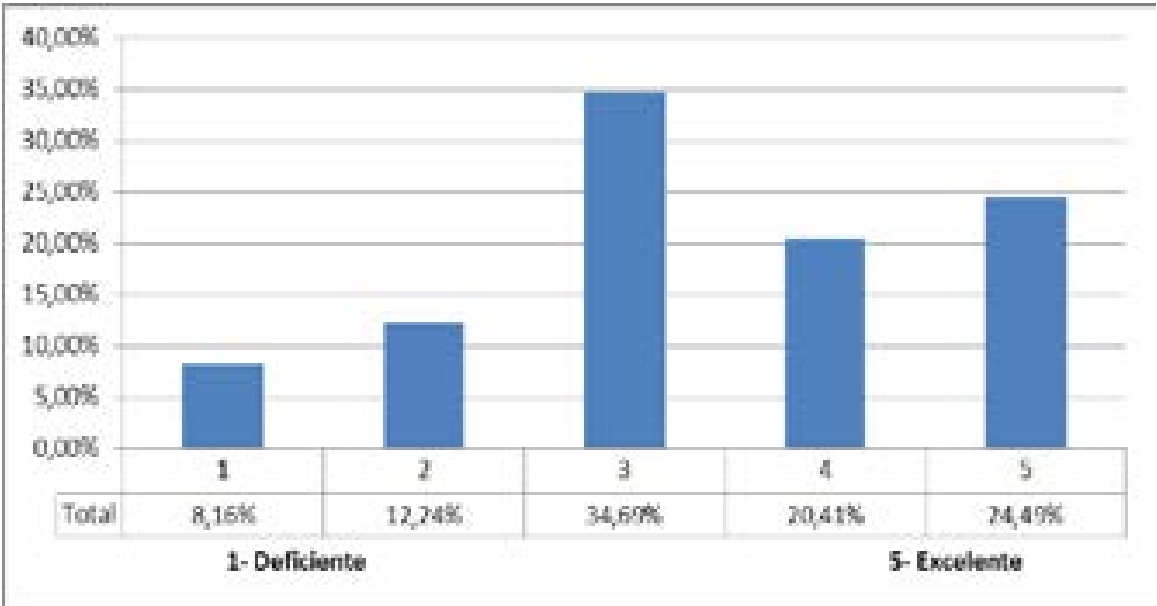

Gráfico 3. Percepción del acceso de las instituciones educativas a los planes que conforman el programa Nacional de Inglés.

Fuente. Elaboración Propia.

Finalmente, en lo referente a la calidad de las capacitaciones, el $51,02 \%$ de los educadores, afirman que el conocimiento aprendido en las capacitaciones puede ser usado en el proceso de enseñanza-aprendizaje con sus estudiantes en el aula. Entre tanto, el porcentaje restante cuestiona la aplicabilidad de los mismos. Esta situación sigue evidenciando la presencia de dificultades en el proceso de implementación del Programa Nacional de Ingles (ver Gráfico 3). 


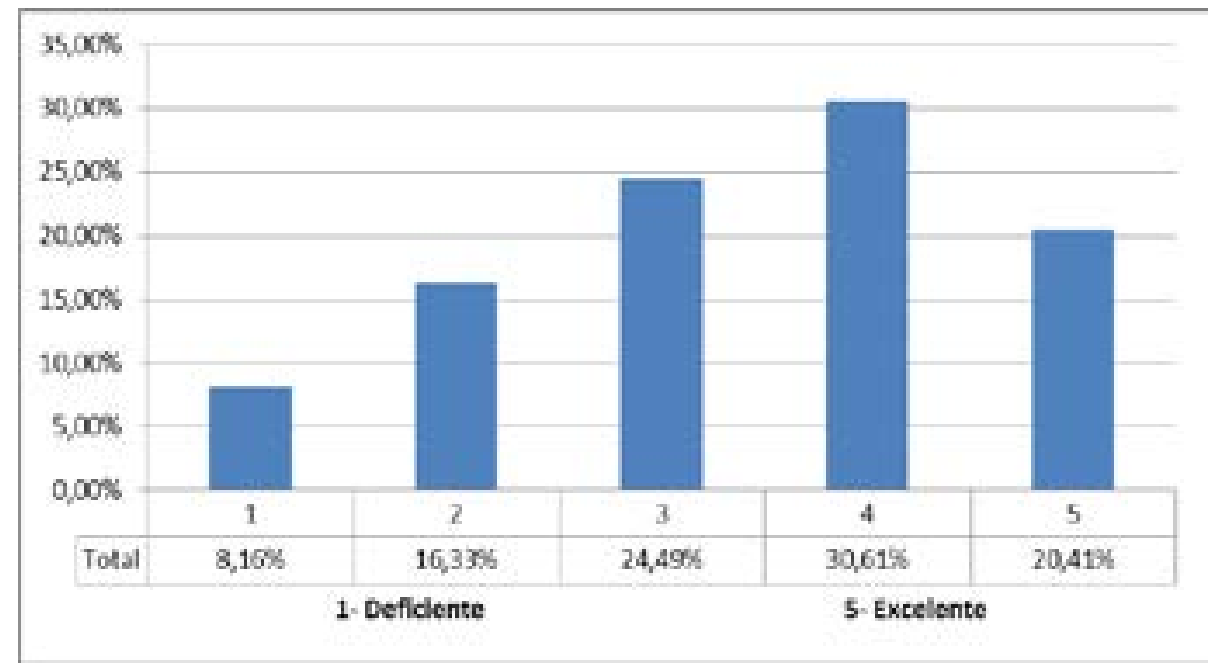

Gráfico 4. Percepción sobre la aplicabilidad de los conocimientos aprendidos en el proceso de enseñanza-aprendizaje

Fuente. Elaboración Propia.

Hasta este punto de la investigación se pudo identificar que un buen número de los docentes del área de Inglés tienen un conocimiento general sobre el Programa Nacional de Inglés frente a su funcionamiento, las articulaciones con las autoridades locales y regionales, así como la posibilidad de tener acceso a cada uno de los beneficios que el plan posee. Cuando se hace énfasis en las capacitaciones, uno de los puntos más importantes del programa, se pudo determinar que las opiniones están divididas en aspectos tales como su funcionamiento, su contenido y la forma en que lo aplican; sin embargo, este también podría ser un indicador de que están haciendo falta capacitaciones, que no se está garantizando la cobertura de los educadores por lo que en la consecución de los objetivos se podría ver afectado en un futuro próximo los resultados esperados, si no se toman algunas medidas para fortalecer el programa en el departamento.

En este aspecto es de resaltar, la percepción positiva que manifiestan el $53,33 \%$ de los docentes encuestados frente a los capacitadores, el dominio de los temas y la capacidad

pedagógica; sin embargo, el porcentaje restante identificaron diversos aspectos a mejorar dentro de los que se destacan: el material usado durante las charlas y su aplicabilidad, convirtiendo este indicador en una apuesta a la que se debería hacerle seguimiento teniendo en cuenta que el programa busca fortalecer el proceso de enseñanza-aprendizaje y este mejorará en la medida en que los docentes tengan las herramientas y apliquen los nuevos conocimientos en las aulas.

De forma complementaria, se identificó falta de socialización sobre el contenido y programa de capacitación, así como poca integración de los docentes, durante el proceso de estructuración de las mismas, proceso que permite conocer las necesidades locales de conocimiento $y$ actualización en el idioma. Otro aspecto a resaltar es el inconformismo con el acceso a infraestructura tecnológica y/o física que sirve de apoyo al proceso de enseñanza - aprendizaje del idioma, teniendo en cuenta que el $24.49 \%$ de los docentes encuestados aseguran que el acceso es deficiente. Estos resultados se convierten en evidencia que el programa se ha enfocado más 
en el plan de capacitaciones, dejando de lado el acceso a herramientas que puedan ayudar a los docentes no solo a fortalecer los procesos de enseñanza-aprendizaje, sino también la actualización y mejoramiento de su nivel de dominio del idioma.

A nivel general se puede observar que el Programa Nacional de Inglés 2015-2025 tiene falencias dentro de su ejecución en el Departamento de Norte de Santander, dado que los docentes no se sienten del todo satisfechos con los procesos y dentro de las instituciones educativas falta mayor socialización y atención para asegurar el éxito del mismo.

\section{Formulación de estrategias desde la gestión pública que puedan aplicarse al Plan Nacional de Inglés 2015-2025 en el departamento de Norte de Santander.}

Una política pública sigue un ciclo de forma sistémica e integral compuesta por unas etapas contexto, contenido, actores y procesos. Cuando se habla de obstáculos de procesos se refiere a la forma en cómo se lleva a cabo la política pública, la institucionalización de los procesos, los canales de interacción/comunicación entre los diferentes niveles, la retroalimentación, el seguimiento y a evaluación de la política y al cronograma de acción (Ortegón Quiñones, 2008). Por esta razón son tan importantes la realización de ejercicios que permitan verificar el desarrollo de las políticas públicas y así formular estrategias que permitan ajustar el rumbo de las mimas.

Una de las herramientas más usadas para la consecución de estrategias es el método DOFA; luego de hacer la valoración de los aspectos evaluados se procede a la definición de estrategias que permitan minimizar las debilidades mientras se potencializan las fortalezas y oportunidades, de este modo se construye la matriz de estrategias y acciones

(Ortegón Quiñones, 2008), que en este caso se pueden utilizar para mejorar los procesos del programa en el departamento de Norte de Santander.

Como resultado de la matriz de estrategias DOFA se establecen unas acciones que permiten optimizar la ejecución del programa en los niveles educativos y gubernamentales con acciones encaminadas a cada uno de los actores del proyecto, mejorando así la gestión pública a través de estrategias que pueden aplicarse para asegurar al máximo la consecución de los objetivos trazados. A continuación, se presentan algunas acciones que se derivan de este trabajo investigativo:

- Establecer una planificación coordinada entre los gobiernos regionales, locales y las instituciones educativas para optimizar la operatividad del programa en el departamento.

- Establecer herramientas de seguimiento y control que permitan mejorar los procesos de forma continua, al mismo tiempo que se reduce al máximo la incertidumbre minimizando al máximo los riesgos.

- Establecer de forma coordinada herramientas de comunicación formal entre los diferentes actores del programa que permitan mejorar el flujo de información y permitan conocer los avances, retos y dificultades que se van presentando durante la ejecución del programa.

- Establecer herramientas de gestión a nivel gubernamental y dentro de las instituciones educativas que permitan evaluar los avances, hacer seguimiento a las acciones desarrolladas y los logros obtenidos, así como eficacia en la ejecución del programa.

- Establecer indicadores de gestión para evaluar la intervención del modelo pedagógico y su eficacia dentro de las instituciones educativas del departamento. 
- Conformar un Comité Técnico en el que se integren a los docentes, con el objetivo de analizar los indicadores de gestión, evaluar los avances y determinar acciones que permitan el logro de los objetivos planteados conforme se va desarrollando el programa.

- Mejorar el acceso a infraestructura física y tecnológica que permita potenciar los procesos de auto aprendizaje de los docentes, perfección de su nivel de inglés así como optimizar los procesos de enseñanza-aprendizaje a través de herramientas educativas basadas en tecnología

- Aprovechar el dominio de temas de los capacitadores para aumentar el número de capacitaciones, la concurrencia y la profundidad de los temas, permitiendo mejorar el nivel del idioma en los docentes así como brindar nuevas herramientas que permitan innovar dentro del aula.

\section{CONCLUSIONES}

A través del desarrollo de esta investigación se identificó la percepción general de los docentes del área de Inglés, del Departamento de Norte de Santander, sobre el Programa Nacional de Inglés 2015 - 2025 y el acceso a los diferentes planes del mismo. Esto permitió determinar los puntos de atención que deben tenerse en cuenta a la hora de continuar con la gestión de la política pública con la que se pretende asegurar el éxito del plan dentro de las instituciones.

Los educadores poseen un buen conocimiento general del programa, sin embargo, tienen sus reparos frente a los procesos de capacitación por su duración, contenido y las herramientas que brinda todo ello orientado al fortalecimiento de los procesos de enseñanza-aprendizaje dentro de las aulas de clase, reconociendo el conocimiento de los capacitadores y su capacidad pedagógica. En contraste, se identificó inconformismo por el acceso a infraestructura física y tecnológica, reconociendo las ventajas que podrían tener el uso de tecnología dentro de sus procesos de mejoramiento y en la enseñanza del idioma con sus estudiantes.

De manos de la percepción de los docentes se pudo construir una matriz que permitió identificar las debilidades y fortaleces del programa en el departamento. Se resalta la disposición y la apropiación de los educadores para asumir el reto planteado por el programa aceptando la inserción en el Proyecto Educativo Institucional y adoptándolo como propio para así lograr la consecución de los objetivos. Sin embargo, se reconocen falencias en el acceso a infraestructura, tecnología y capacitaciones; estas últimas parecen ser insuficientes, con temas que no llenan del todo las expectativas de los docentes y cuya duración también es puesta en duda por los educadores. Pero sin duda una de las amenazas más fuertes que presenta el programa es la interoperabilidad entre las instituciones educativas y las autoridades gubernamentales de ámbito regional y local, teniendo en cuenta que el trabajo conjunto es la principal herramienta para conocer cómo va el proceso, los retos y las necesidades que se poseen con miras a cumplir la meta del mejoramiento del nivel de inglés de, al menos, $50 \%$ de los estudiantes en las Pruebas Saber 11 del año 2025.

De igual forma se establece que dentro de las instituciones educativas también hace falta un trabajo coordinado que permita la socialización de las capacitaciones entre docentes y las autoridades de las mismas, con el objeto de que los educadores beneficiados puedan aportar los conocimientos adquiridos con los docentes no beneficiados del programa, al mismo tiempo que se planean y llevan a cabo estrategias conjuntas para mejorar el proceso de enseñanzaaprendizaje del inglés dentro de sus instituciones educativas. Por otro lado, también se evidencia 
que los educadores pueden no estar conformes con los contenidos y herramientas suministradas en las capacitaciones en parte debido a la poca integración del cuerpo docente a la planeación de las mismas, con el objeto de identificar las necesidades locales que pueden diferir de las de los docentes de otras regiones del departamento y del país.

Finalmente, a través de la matriz DOFA se definieron unas estrategias encaminadas en el mejoramiento de la gestión pública del Programa Nacional de Inglés 2015- 2025 en el Departamento de Norte de Santander; estas se formularon teniendo en cuenta las fortalezas, oportunidades, amenazas y debilidades del programa, con el objeto de minimizar al máximo el actual riesgo y fortalecer las ventajas operativas que actualmente tiene el programa.

Sin embargo, se limitó a su formulación teniendo en cuenta que el diseño, estructuración e implantación de las estrategias de gestión pública para el programa, deben contar con el acompañamiento de los actores del mismo, lo que permite tener en cuenta todas las perspectivas para atender de la mejor forma las problemáticas existentes y así lograr el objetivo común de mejorar los procesos y optimizar el funcionamiento del Programa Nacional de Inglés 2015-2025 en el departamento.

\section{REFERENCIAS BIBLIOGRÁFICAS}

Asamblea Nacional Constituyente. (1991). Constitución Política de Colombia.

Baker, C. (2001). Foundations of bilingual education and bilingualism (3 ed.). Multilingual Matters Ltd.

Bejarano, P.A. (2013). Propuesta metodológica para la enseñanza del inglés a través de la Bilingüismo en Algunas Instituciones Públicas:
Factores Lingüísticos

y Pedagógicos.

Recuperado de:

http://www.scielo.org.co/pdf/

calj/v13n2/v13n2a06.pdf

Calderón. \& Mora, Y. (2012). Formación permanente del docente de inglés: una

Campo, M.F. (2014). Colombia Very Well! Programa Nacional de Inglés (Ministerio Educación, 2014).

Fishman, J. A. (2002). El nuevo orden lingüístico. UOC Digit. HVM revista digital dé hummanitats.

Recuperado de: https://www.uoc.edu/ portal/es/index.html/articles/esp/ fishman/fishman_imp.html

Gobernación de Norte de Satander. (2019, 05 24). Retrieved from http://www. nortedesantander.gov.co/NoticiasGobernaci\%C3\%B3n-Norte-deSantander/ArticleID/12834/Inicia-elproyecto-de-biling\%C3\%BCismo-enlas-instituciones-educativas-de-Nortede-Santander.

Gómez, R. (2012). Gestión de políticas públicas: aspectos operativos, Rev. Fac. Nac. Salud

Graddol, D. (1997). The future of English. The British Council. Recuperado de Graw-Hill.

Hernández, R., Fernández, C. \& Baptista P. (2014).

Metodología de la Investigación. 6ta http://wsp.presidencia.gov.co/ Normativa/Leyes/Documents/ 2013/LEY\%201651\%20 DEL\%2012\%20DE\% 20JULIO\%20DE\%202013.pdf http://www.praxisinvestigativa. mx/assets/12_7_la_ensenanza_del_ ingles.pdf 
http://www.scielo.org.mx/scielo.php?

script=sci_arttext\&pid=

S1405-66662016000200385

https://bibliotecadigital.

univalle.edu.co/bitstream/handle/

10893/9095/CB-0510978.pdf;

jsessionid=24ED5639656FFEAED8

E5A40D192BFF85? sequence $=1$

https://www.academia.edu/12529756/

The_Future_of_English_

by_David_Graddol

https://www.corteconstitucional.gov.co/

inicio/Constitucion\%20politica\%

20de\%20Colombia.pdf

https://www.mineducacion.gov.co/

1621/articles-115174_archivo_pdf.pdf

https://www.mineducacion.gov.co/

1621/articles-85906_archivo_pdf.pdf

https://www.uoc.edu/portal/es/i

ndex.html/articles/esp/fishman/

fishman_imp.htmlhummanitats.

Recuperado de:

Kachru, Y. \& Nelson, C. L. (2011).

World Englishes in Asian Contexts.

China: Hong Kong

Maturana, L. M. (2011).

La Enseñanza del Inglés en

Tiempos del Plan Nacional de

Ministerio de Educación Nacional.

(2006).

Estándares básicos de

competencias en Lengua

Muñoz, M. (2015).

La enseñanza del inglés

en planes y programas de

estudio de las

Murray, R. S., \& Larry, J. S. (2009).

Estadística. 4ta edición. México DF, México: Mc

Ortegón Quiñones, E. (2008).

Guía sobre diseño y

gestión de política pública. Bogotá:
Organización del Convenio Andrés Bello,

Conciencias, Instituto de

Estudios Latinoamericanos.

pedagogía por proyectos.

Recuperado de:

personas jóvenes y adultas. Recuperado de:

Pública 2012,30(2), 223-236.

Recuperado de:

Ricoy, M.C., \& Álvarez, P. S. (2016).

La enseñanza del inglés en la

educación básica de

Royo, J. (2000). Argumentos para el Bilingüismo.

España: Novagrafik,

Serrano, S. (2024). Plan de Desarrollo para

Norte de Santander 2020-2023.

Recuperado del

sitio de internet de la gobernación de

Norte de Santander:

http://www.nortedesantander.gov.co/

portals/O/PDD_2020-2023_

Plan_de_desarollo.pdf

sitio de internet del Ministerio de

Educación Nacional:

https://www.mineducacion.gov.co/

1759/articles-343837

Programa_Nacional_Ingles.pdf

Tamayo y Tamayo, (2014),

El proceso de investigación científica.

México: Editorial Limusa. University Press. 